\title{
Spinal and Femoral Bone Mineral Density in Patients with Sexual Precocity, Delayed Adolescence and Hypogonadism: Comparison With Normal Controls
}

\author{
Yoshihito Takahashi, Kanshi Minamitani, Yasuyuki Kobayashi, Masanori Minagawa, Toshiyuki Yasuda \\ and Hiroo Niimi
}

Department of Pediatrics, Chiba University School of Medicine, Chiba, Japan

Key words: bone mineral density, peak bone mass, sex steroids, dual energy x-ray absorptiometry.

\section{Introduction}

We determined when bone mass of the lumbar spin (L2-L4) $\left(\mathrm{g} / \mathrm{cm}^{2}\right)$ and femoral neck $\left(\mathrm{g} / \mathrm{cm}^{2}\right)$ reaches its peak in healthy Japanese, and examined the influence of exposure to sex steroids on bone mineral density (BMD). Also we determined the volumetric $\mathrm{BMD}$, termed bone mineral apparent density (BMAD), of the lumbar spine and femoral neck.

\section{Subjects and Methods}

To examine the influence of exposure to sex steroids on bone mineral density (BMD), we measured the BMD of both the lumbar spine and femoral neck in 11 patients with female central sexual precocity, and three with female primary hypogonadism 5 with delayed adolescence, by dual-energy $x$-ray absorptiometry (DXA) (Hologic QDR-1,000). Normal BMD was obtained by measuring the BMD of 31 healthy children aged 2-11 yrs, 269 children (138

Correspondence: Dr. Yoshihito Takahashi, Department of Pediatrics, Chiba University School of Medicine, 1-8-1, Inohana, Chuo-ku, Chiba 260 Japan males and 131 females) aged 13-19 yrs, and 12 males and 12 females aged 20-34 yrs as adult controls. Because the densitometric data obtained from DXA are strongly influenced by the size of the bone in growing subjects, the volumetric $\mathrm{BMAD}\left(\mathrm{g} / \mathrm{cm}^{3}\right)$ of the vertebral cube (L2-L4) and femoral neck were determined: $\operatorname{BMAD}\left(\mathrm{g} / \mathrm{cm}^{3}\right)=\operatorname{BMD}\left(\mathrm{g} / \mathrm{cm}^{2}\right) / \sqrt{ }$ scanned area $\left(\mathrm{cm}^{2}\right)$ for the lumbar spine, and BMAD = $\mathrm{BMD} /$ width for the femoral neck.

\section{Results and Discussion}

The BMD, in both lumbar spine and femoral neck, nearly reached its peak at age 14.5-15 yrs in females and 16.5-17 yrs in males. In patients with sexual precocity it was high compared to age-matched controls, whereas patients with primary hypogonadism and delayed adolescence showed lower lumbar apparent $\mathrm{BMD}$, and an increase in lumbar $\operatorname{BMAD}\left(\mathrm{g} / \mathrm{cm}^{3}\right)$ was noted after the progression of puberty in healthy children, probably suggesting the importance of sex steroids in the increase in BMD and lumbar BMAD in both sexes. 


\section{Takahashi et al.}

We conclude that BMD is crucially affected by the sex steroids and that a further longitudinal study is required to achieve a high peak bone mass in these patients.

\section{References}

1. Takahashi Y, Minamitani K, Kobayashi $\mathrm{Y}$, Minagawa M, Yasuda T, Niimi $\mathrm{H}$. Spinal and femoral bone mass accumulation during normal adolescence: Comparison with female patients with sexual precocity and with hypogonadism. J Clin Endocrinol Metab 1996; 81: 1248-53. 